Case Report

\title{
Replasing Polychondritis and Geriatrics: Report of Two Cases
}

\section{Nassima Dekdouk*, Djennette Hakem, Lakhder Amine Betaimi, Djamel Eddine Benkali, Taous Hadded, Abdelkrim Berrah}

Department of Internal Medicine, Faculty of Medicine, Benyoucef Benkhedda University, Algiers, Algeria

\author{
Email address: \\ ness.popouna@gmail.com (N. Dekdouk) \\ ${ }^{*}$ Corresponding author
}

\section{To cite this article:}

Nassima Dekdouk, Djennette Hakem, Lakhder Amine Betaimi, Djamel Eddine Benkali, Taous Hadded, Abdelkrim Berrah. Replasing Polychondritis and Geriatrics: Report of Two Cases. American Journal of Internal Medicine. Vol. 6, No. 2, 2018, pp. $29-33$.

doi: 10.11648/j.ajim.20180602.11

Received: March 5, 2018; Accepted: April 2, 2018; Published: May 17, 2018

\begin{abstract}
We report two cases of geriatric replasing polychondritis [RP] observed in internal medicine; and the problems posed by the therapeutic management of this condition at this phase vulnerable of life. The first case was a70- years old women without antecedents, presents a diffuse and painful and bilateral increase of both ears, and painful red eyes with blurring of vision. The set of disorders evolves in a context of fever and deterioration of the general state and poly arthralgia of mixed schedule. The objective clinical examination finded a deformed ears 'Cauliflower ear'; and a breath of aortic insufficiency in cardiac auscualtation; and bilateral episcleritis in the tow eyes objective in ophtalmic examination; associated with a biological inflammatory syndrome; the echocardiography objective, the moderate aortic regurgitation. The second case was a67 year old male presented,with a history of pain and swelling of both ears lobes, with recurrent red left eye painless.He has a history of type 2 diabetes and hypertension, clinically he was noted to have a swollen exquisitely tender erythematous upper cartilaginous part of the right pinna with sparing of the earl obule,episcleritis in the left eye in a ophtalmologic examination associated a frank inflammatory syndrome, the echocardiography objective a mild oartic regurgitation. The diagnosis of RP is retained according to the criteria of michet et al in both patients. The 2 patients evolve favorably under corticotherapy instituted at a rate of $1 \mathrm{mg} / \mathrm{Kg} /$ day.Corticotherapy treatment exposed the patient (case 1) to the appearance of osteoporosis with high risk of fracture and the appearance of controllable hypertension under treatment; and the patient (case 2) at imbalance of his hypertension and diabetes which led us to change his therapeutic arsenal to avoid metabolic and cardiovascular complications. The rarity of the disease and the variability of its clinical spectrum explain the lack of a therapeutic trial controlled and empirical nature of the therapeutic recommendations. Evolution is by pushing successive, whose frequency and severity are extremely variable. Complications Cardiovascular diseases are common and responsible for the death of one out of four patients, which clinician to screen them so as not to delay a sometimes difficult treatment.
\end{abstract}

Keywords: Replasing Polychondrotisis, Geriatric, Comorbidities

\section{Introduction}

Relapsing polychondritis is a rare autoimmune rheumatic disorder characterized by episodic inflammation of cartilaginous tissue throughout the body [1-27].The disease can affect several organs, including proteoglycan rich tissues, particularly ears,nose, respiratory tract, eyes, and joints.

The first to describe the disease was Jaksch- Wartenhorst from the Faculty of Medicine of the German University in Prague, in 1923, who called it polychondropathy [2, 3]; the term "relapsing polychondritis" was first used by Pearson et al. In 1960 in their review of 12 cases [4].

The rarity of RP makes epidemiological data very scarce. Its incidence is poorly documented and probably underestimated. An estimated incidence of 3.5 cases per million people has been reported in a study in Rochester 
(Minnesota, USA) [5-7] in a recent UK study, the prevalence of RP was 9.0 (95\% confidence interval (CI) 7.6e10.5) per million and its incidence was $0.71(95 \%$ CI $0.55 \mathrm{e} 0.91)$ per million per year [1]

The peak age at disease onset is the fifth decade of life and appears to occur with equal frequency in both sexes; but RP may appear during childhood and as late as the eighth decade $[1,5-7]$

It is believed to be an immunologic reaction to collagen Type II which is predominantly presented in the cartilaginous structures of the body and in the eye $[8,9]$.

\section{Materials and Methods}

\subsection{Illustrative Case Reports}

Case 1.

A 70 -year-old woman was consulted in our department for recurrent swelling of both ears, arthralgia of the large joints of the lower limbs, and painful red eyes with blurring of vision. All disorders evolve in a context of irregular fever up to $39^{\circ} \mathrm{C}$ and alteration of the general state. Her personal and family history was unremarkable.

During physical examination, the patients' ears were deformedred, swollen, and tender, 'Cauliflower ear': the normal relief outlines have disappeared. The external auditory canal has collapsed, and its opening can no longer be seen, but the non cartilaginous lobule is spared (figure 1.A). Cardiac auscultation finded a breath of aortic insufficiency was finded, the rest of the clinical examination is without abnormalities; Ophthalmologic examination showed episcleritis in the tow eyes (figure 1.B).

Routine blood investigations revealed normocytic normochromic anemia, elevated erythrocyte sedimentation rate. The rheumatoid factor was within normal limits. Other clinical parameters (urinalysis, thyroid tests, and liver function tests) resulted within normal range. Antinuclear antibodies (ANA) titer was 1: 320. Antiphospholipid antibodies, antineutrophil cytoplasmic antibodies (ANCAs), anti Borrelia burgdorferi antibodies $\operatorname{IgG} / \mathrm{IgM}$, rheumatoid factor, anti-HIV-1, anti-HIV-2, and VDRL tests were negative.

Chest radiography; EKG without anomaly

Echocardiography showed a moderate aortic régurgitation.
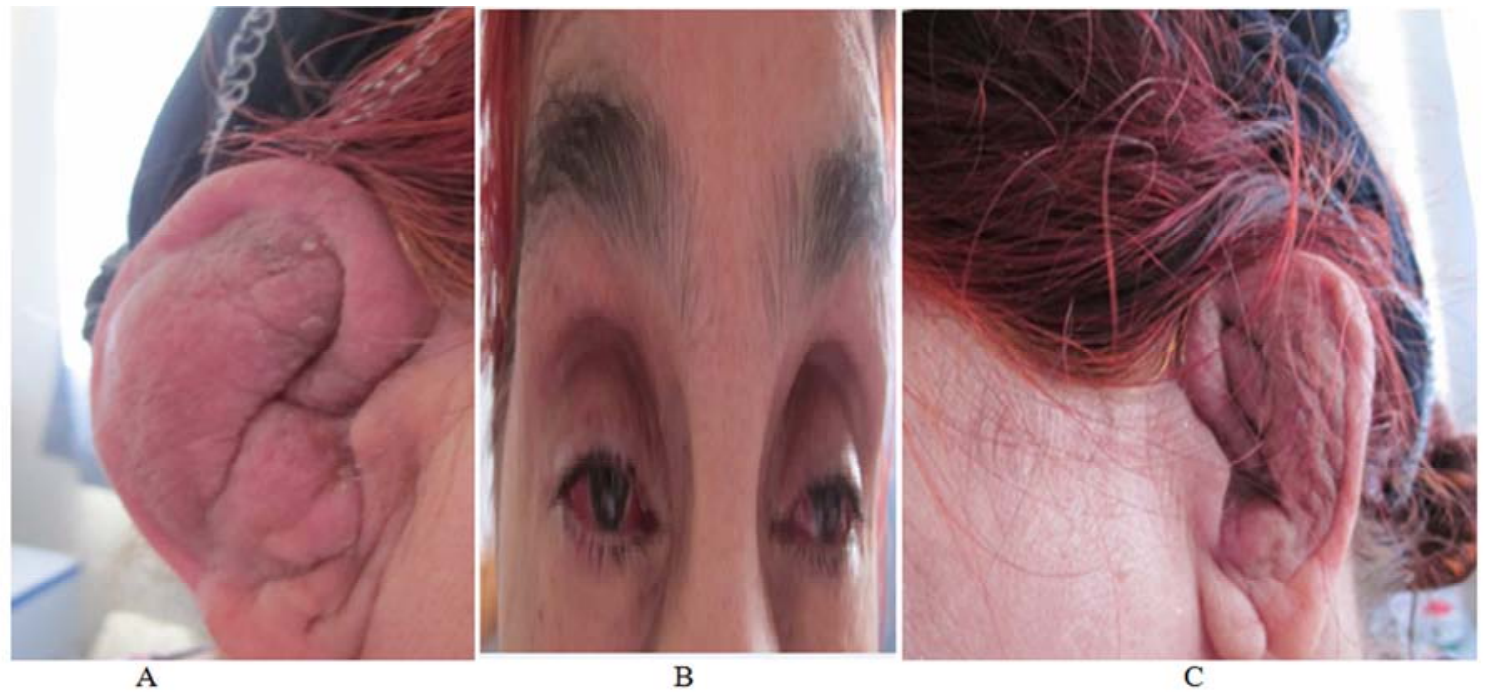

Figure 1. A. 'Cauliflower ear': the normal relief outlines have disappeared and the ear appears thickened and red, swollen. The external auditory canal has collapsed, and its opening can no longer be seen, but the non cartilaginous lobule is spared. . B. Episcleritis. c. Evolutionthe auricular chondritis after 10 days of traitement. Disappearance of swelling of the ear with release of the external auditory canal.

\section{Case 2.}

A 67-year-old male presented with a history of pain and swelling of both ears lobes, with recurrent red left eye painless.

The patient is a known hypertensive and diabetic, for which he has been receiving treatment for the past 15 years.

Physical examination showed the patient to be in regular general condition, slimmed, eupneic, $\mathrm{HR}=94$, arterial blood pressure $11 / 8 \mathrm{~cm}$ of $\mathrm{Hg}$. he was noted to have a swollen exquisitely tender erythematous upper cartilaginous spart of the right pinna with sparing of the ear lobule (figure 2.A). There was no alteration in the heart, nor in the abdomen. Ophthalmologic examination showed episcleritis in the left eye (figure 2.B).

\section{LABORATORY FINDINGS}

Creatinin $=10 \mathrm{mg} / 1$; creatinin clearance $=80 \mathrm{ml} / \mathrm{min} / 1.73 \mathrm{~m}$; Potassium $=4 \mathrm{mEq} / 1$; sodium $=138 \mathrm{mEq} / 1 ; \mathrm{GOT}=14 \mathrm{UIl}$, GPT $=14 \mathrm{U} / \mathrm{l} ;$ Erythrocyte count $=4.68 \times 10 \mathrm{I} \mathrm{mm} 3 ; \mathrm{Hb}=13 \mathrm{~g} / \mathrm{dl} ; \mathrm{Ht}$ $=38 \% ; \mathrm{MCH}=27.73 \mathrm{pg} ; \mathrm{MCHC}=30 \mathrm{~g} / \mathrm{dl} ; \mathrm{MCV}=82$; platelet $=260,000 ;$ leukocyte $=9800 ; \mathrm{Alb}=3.1 \mathrm{~g} / \mathrm{dl}$; Urinalysis: $\mathrm{pH}=6.5, \mathrm{D}=1020$, prot (-), gluc(-), leukocytes $=$ $12,000 / \mathrm{ml}$, erythrocytes $=800,0001 \mathrm{ml}$ dismorphism $(-)$.

Antinuclear antibody (-), anti native DNA antibody (-), anti ENA antibody (U1-RNP, anti-Sm, anti SS-AfRo, anti-SS-B/La); Rheumatoid factor (-); cryoglobulin (-); anticardiolipin antibody (-); ANCA (-);

$\mathrm{C}$ reactive protein $=1 / 16 ;$ LE cels $(-) ;$ anti-HIV-1, anti-HIV-2,HVB et HVC and VDRL tests were negative; sedimentation rate $=60(1-7 \mathrm{~mm} / \mathrm{h}) ;$ Prothrombin Time $=100$ $(>70 \%)$; RNI $=1$; PTT $=29$ ' 
Chest radiography; EKG were normal.

Echocardiography showed a mild aortic régurgitation.

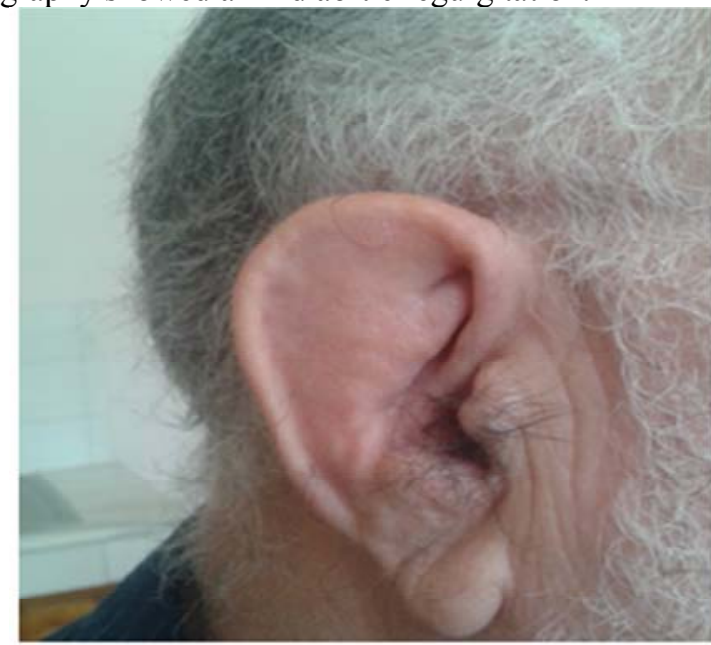

A

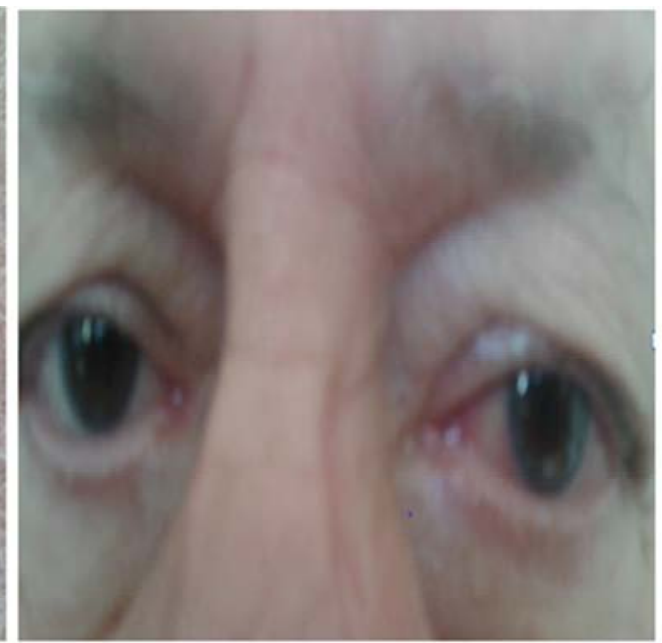

B

Figure 2. A. Auricular chondritis in a patientwith with relapsing polychondritis. Note the soft lobule is separed;B. Diffuse scleritis in a patient with relapsing polychondritis.

\subsection{Management and Out-Come}

The diagnosis of RP was retained in both patients according to the criteria of Michet et al: Inflammation in the auricular cartilage, episcleritis, Seronegative polyarthritis.

The 2 patients evolve favorably under corticotherapy instituted at a rate of $1 \mathrm{mg} / \mathrm{Kg} /$ day, (figure 1.C).Diabetes and hypertension are well controlled - in the short and medium terms - under drastic diet regimes and with the addition of insulin therapy (case2).There is no short or medium term neuropsychiatric disorders, but there is anorexia (case 1) with increased vitamin deficiencies requiring protein and vitamin-calcium supplementation.

\section{Discussion}

The diagnosis of RP rests on clinical grounds and is frequently delayed [6]. Michet et al. developed criteria for diagnosing RP [5-7, 10-12]. An advantage of their criteria is that a biopsy does not need to be obtained routinely [10]; Al though not validated, the criteria of Michet et al. are useful in clinical practice.

There is growing evidence in the literature that relapsing polychondritis is an immunologic reaction to collagen Type II $[5-7,11,13]$ which is predominantly present in the cartilaginous structures of the body and in the eye. Almost one third of patients with RP exhibit circulating antibodies to type II collagen during the acute phase of the disease $[5,6,8,11,14]$.

Specific antibodies against type II collagen, primarily IgG, were detected in the serum of $50 \%$ of patients with RP [2]; however, it should be noted that these antibodies are not RP-specific and were identified also in rheumatoid arthritis (RA) [5]; they are directed against various epitopes of the collagen molecule. [14]

RP is generally observed in the fourth and fifth decades of life and occurs with equal frequency in both sexes but geriatric onset forms have been described [1, 5, 6, 15].

The clinical picture of the RPC changes with severity and duration of the disease.

The Inflammation and destruction of the auricular cartilage, non erosive arthropathy, and nasal collapse are the most common features of relapsing polychondritis $[5-7,11,13$, 15-17] Other structures that maybe affected include the eyes, cochlear or vestibular structures, cardiac valves, large vessels, and the kidneys, skin Neurologic abnormalities, Vasculitis, Other symptoms, such as fatigue and weight loss, are common in RP and fever often accompanies the acute stages of the disease...[5-7, 11-13, 15, 18-24].

The ocular manifestations are rarely inaugural but develop eventually in $60 \%$ of patients [9-11, 18, 24]. They are usually mild, consisting in conjunctivitis or, more often, unilateral or bilateral episcleritis and/or scleritis [24].

Cardiac complications are more prevalent in older and male patients and are the second cause of death in RP [5, 25] appear in about $27 \%$ of patients with RP [6, 7] and include aortic and mitral regurgitation, aortic aneurysm[5, 11, 23, 25], aortic dissection, myocarditis, pericarditis, myocardial infarction /angina pectoris [25]; atrioventricular block, and systemic vasculitis [26]. The Aortic valve incompetence is a feature in $10 \%$ of patients with RP [10]. However, the comorbidities in RP have been scarcely described in the literature. Notably, tow studies, reporting the incidence, and prevalence of cardiovascular diseases and their risk factors( Arterial hypertension, Diabete smellitus, Dyslipidemia; Cardiac insufficiency, Myocardial infarction, Stroke, old age ) [1, 27].

The existence of several clinical phenotypes has been recently reported: the haematological form associated with myelodysplasia affecting less than $10 \%$ of patients, mainly elderly men with poor prognosis [7]. The respiratory form affecting about $25 \%$ of patients with predominant 
tracheobronchial involvement and clean complications [7, 11], and the benign, most common, $65 \%$ of patients, good prediction.

Mortality in RP is more than twice of the general population [1] and the most frequent causes of death are respiratory disease, heart conditions and cancer[1, 7, 19, 27]; The most cases concluded that there is an increased relative risk of malignancy in RP with old age [22].

The treatment regimen is tailored empirically to each individual patient , based on clinical activity and severity [10, 13]; Corticosteroids and cyclophosphamide can decrease the frequency, duration, and severity of recurrences, but might not be able to stop the disease progression $[13,26]$

The use of dapsona, colchicine anti-CD4 monoclonal antibody, D-penicillamine and antimalarials" has also been described $[5,7,10-13,15,20]$.

Criteria for diagnosing relapsing polychondritis $[6,7,10$, 12].

\section{Major criteria}

Inflammation in the auricular cartilage

Inflammation in the nasal cartilage

Inflammation in the laryngo-tracheal cartilage

Minor criteria

Conjonctivitis ,episcleritis ,scleritis, oruveitis

Hearing loss

Vestibular dysfunction

Seronegative polyarthritis

Two major criteria or one major criteria+two minor criteria are needed to classify the patient as having relapsing polychondritis.

\section{Conclusion}

The rarity of the disease and the variability of its clinical spectrum explain the lack of a controlled therapeutic trial and the empirical nature of the therapeutic recommendations. These recommendations also ignore the specificity of this age-related affections ; The recommended therapies do not take into account the co-morbidities often associated in geriatric forms and cause major iatrogenic complications (infection, ketosis, psychiatric disorders, osteoporotic fractures, etc.). The evolution is done by successive shoots whose frequency and severity are extremely variable. Cardiovascular complications are frequent and responsible for the death of one in four patients, which requires the clinician to screen them so as not to delay a sometimes difficult treatment.

\section{References}

[1] Nisha Hazraet al.Incidence and mortality of relapsing polychondritis in the UK: a population-based cohort study; Rheumatology 2015;54:2181-2187.

[2] Rovenský J, Sedláčková M (2016) Relapsing Polychondritis. J Rheum Dis Treat 2:043.
[3] H. Rhys Davies and A.R.Kelsall. Atrophic polychondritis with the report of a case;Ann. rheum. Dis. (1961), 20, 189.

[4] Beata Sosada, KatarzynaLoza, and EwelinaBialo-Wojcicka. Relapsing Polychondritis.Case Reports in Dermatological Medicine Volume 2014, Article ID 791951,4 pages.

[5] Mathian A, et al., Relapsing polychondritis: A 2016 update on clinical features, diagnostic tools, treatment and biological drug use, Best Practice \&Research Clinical Rheumatology (2016).

[6] L. Cantarini et al. / Journal of Autoimmunity 48-49 (2014) 53-59.

[7] Dion J, et al Relapsing polychondritis: What'snewin2017?; Rev Med Interne(2017); [Article in French].

[8] Abdul Latif Hamdan, DojaSarieddine. Larngeal Manifestations ofRelapsingPolychondritis; Open Journal of Rheumatology and Autoimmune Diseases, 2013, 3, 108-112.

[9] T. Lahmer et al. Relapsing polychondritis: An autoimmune disease with many faces;Autoimmunity Reviews 9 (2010) 540-546.

[10] Puéchal X, et al. Relapsing polychondritis. Joint Bone Spine (2014).

[11] Longo L, et al, Relapsing polychondritis: A clinical update, Autoimmun Rev (2016).

[12] Sabine Schumacher, Herwig Pieringer; Relapsing polychondritis: a chameleon among orphan diseases; Wien Med Wochenschr (2017).

[13] Antonio Vitale, et al. Relapsing Polychondritis: an Update on Pathogenesis, Clinical Features, Diagnostic Tools, and Therapeutic Perspectives ;Curr Rheumatol Rep; (2015).

[14] Letko et al. Relapsing Polychondritis: A Clinical Review; Seminars in Arthritis and Rheumatism, Vol 31, No 6 (June), 2002: pp 384-395.

[15] Tyng Yu Chuah, Nai Lee Lui; Relapsing polychondritis in Singapore: a case series and review of literature; Singapore Med J 2017; 58(4): 201-205.

[16] Emmungil and Aydin. Relapsing polychondritis; Eur J Rheumatol 2015; 4: 155-9.

[17] JibahEng, FRCS, and Sabaratnam Sabanathan, FRCS. Airway Complications in Relapsing Polychondritis; Ann Thorac Surg 1991:51:686-92.

[18] Bradley L,et al. Ocular and Systemic Findingsin Relapsing Polychondritis; Ophthalmology 93:681-689, 1986.

[19] Drosos A. A. Relapsing Polychondritis; Orphanet encyclopedia, May 2004.

[20] Miyasaka,LS, et al.Relapsing polychondritis Sao Paulo Medical Journal/RPM 116(1): 1637-1642, 1998.

[21] Jérémie Dion et al. Relapsing polychondritis can be characterized by 3 different clinical phenotypes: Analysis of a recent series of 142 patients; Arthritis \& Rheumatology (2016).

[22] Raida BS, Yosra C, Faten F, Mouna S, Moez J, et al. (2016) A Relapsing Polychondritisand Malignancies: A Case Report and Review of Literature. J Dermatol Res Ther 2:04.

[23] Le Besnerais M, Arnaud L, Bout' emy J, Bienvenu B, L' evesque $\mathrm{H}$, Amoura Z, Marie I, Aortic involvement in relapsing polychondritis,Joint Bone Spine (2017). 
[24] Furuya et al; Relapsing polychondritis with different types ofocular inflammations; International Medical Case Reports Journal 2015:8 193-197.

[25] Jun Shimizu, Hiroshi Oka, Yoshihisa Yamano, Kazuo Yudoh and Noboru Suzuki;Cardiac involvement in relapsing polychondritis in Japan, Rheumatology 2016; 55: 583584.

[26] A-G A Selim, L G Fulford, R H Mohiaddin, M N Sheppard.
Active aortitis in relapsing polychondritis; J Clin Pathol 2001; 54: 890-892.

[27] Pablo Arturo Olivo Pallo, Maurício Levy-Neto, Rosa Maria Rodrigues Pereira, Samuel Katsuyuki Shinjo. Relapsing polychondritis: prevalence of cardiovascular diseases and its risk factors, and general disease features according to gender; rev bras reumatol. 2017; 57(4): 338-345. 